ORNL/TM-2004/299

\title{
RECENT DEVELOPMENTS IN THE MCNP-POLIMI POSTPROCESSING CODE
}

Sara A. Pozzi

November 2004

Prepared by OAK RIDGE NATIONAL LABORATORY

P. O. Box 2008

Oak Ridge, Tennessee 37831

managed by

UT-Battelle, LLC

for the

U.S. DEPARTMENT OF ENERGY

under contract DE-AC05-00OR22725 


\begin{abstract}
The design and analysis of measurements performed with organic scintillators rely on the use of Monte Carlo codes to simulate the interaction of neutrons and photons, originating from fission and other reactions, with the materials present in the system and the radiation detectors. MCNP-PoliMi is a modification of the MCNP-4c code that models the physics of secondary particle emission from fission and other processes realistically. This characteristic allows for the simulation of the higher moments of the distribution of the number of neutrons and photons in a multiplying system. The present report describes the recent additions to the MCNP-PoliMi post-processing code. These include the simulation of detector dead time, multiplicity, and third order statistics.
\end{abstract}




\section{CONTENTS}

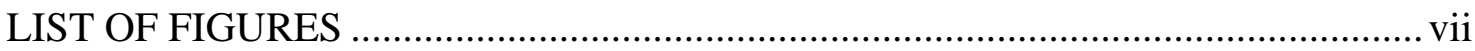

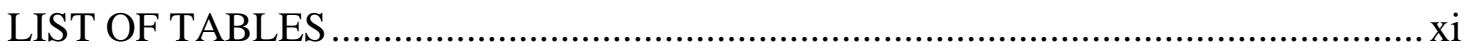

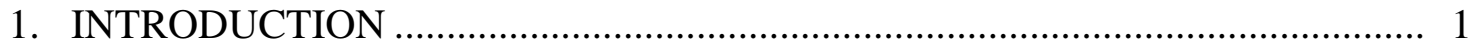

2. SIMULATION OF DETECTOR RESPONSE................................................ 1

3. SIMULATION OF COVARIANCE AND BICOVARIANCE FUNCTIONS ....... 4

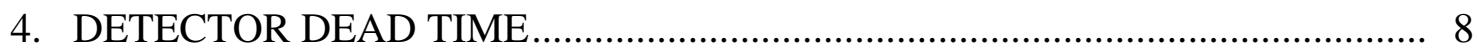

5. SIMULATION OF DETECTOR MULTIPLETS _............................................. 9

6. SUMMARY OF POST-PROCESSING OPTIONS ……………………………..... 11

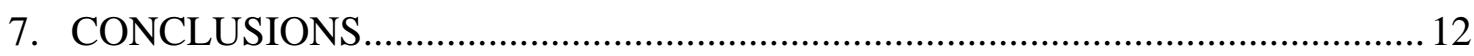

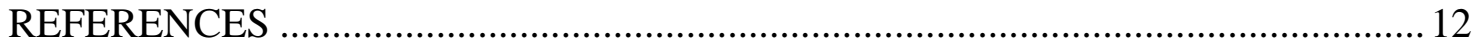

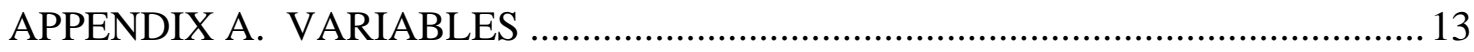




\section{LIST OF FIGURES}

Figure

Page

$1 \quad$ Measured light output functions for plastic (BC 420) and liquid (BC 501) scintillators 3

2 Comparison of Monte Carlo simulation and measurement for a Cf-252 source and detector covariance function.

3 Sketch of the configuration for the simulation of the source-detectors bicovariance function with plutonium sample (not to scale). 6

4 Source-detectors bi-covariance with plutonium sample: (a) Experimental result and (b) MCNP-PoliMi simulation. 7

5 Simulation of source-detectors bi-covariance with plutonium sample: (a) photon photon pairs, (b) neutron - neutron pairs, (c) neutron - photon pairs, and (d) photon neutron pairs.

6 Simulation of source-detectors bi-covariance with plutonium sample: (a) generation zero - generation zero pairs, (b) induced fission - induced fission pairs, (c) generation zero induced fission pairs, and (d) induced fission - generation zero pairs. 8

$7 \quad$ Source-detectors correlation function for $3.3 \mathrm{~kg}$ Pu metal sample and varying detector dead times. 9

$8 \quad$ Illustration of pulse train for multiplet simulation. (a) source-triggered multiplets (b) detector triggered multiplets. 10

$9 \quad$ Source-triggered multiplets for $3.3 \mathrm{~kg}$ Pu metal sample and varying detector dead times. The time window was set to $100 \mathrm{~ns}$ 


\section{LIST OF TABLES}

$\begin{array}{lll}\text { Table } & \text { Page }\end{array}$

$1 \quad$ Excerpt from MCNP-PoliMi output file................................................. 2

$2 \quad$ Input parameters in post-processing code. .............................................. 12 


\section{INTRODUCTION}

This report describes recent enhancements that have been made to the MCNP-PoliMi Matlab $^{\mathrm{TM}}$ post-processing code. The code is used to simulate detection events in liquid or plastic scintillators by post-processing the collision data output from the MCNP-PoliMi simulation. Previous versions of the code were used to simulate time-dependent covariance measurements of the signals from the source and a detector, and from two detectors. The current version of the code includes the simulation of third-order statistics (bicovariance functions) and multiplicity. Detector dead time is also included in the new post-processing code.

The main features of the MCNP-PoliMi code [1] can be summarized as follows. The simulation of spontaneous and induced fission includes the correct multiplicity of prompt neutrons and gamma rays from individual fission events. In addition, the algorithm generating secondary gamma rays from neutron interactions has been changed substantially from the standard MCNP algorithm. Neutron interaction and secondary gamma ray production are now linked to provide a better representation of the physics of individual particle interactions. A number of spontaneous fission and alpha/n neutron and gamma ray sources have been added. The output format of MCNP-PoliMi is in the form of a collision output table, which allows for the simulation of detector response with more flexibility. Further details on the modifications are given in reference 1.

This report is organized as follows. Section 2 describes the MCNP-PoliMi output file and the simulation of organic scintillator detector response. The simulation of the covariance and bicovariance functions is discussed in Section 3, and Section 4 describes the implementation of detector dead time. The simulation of multiplets is discussed in Section 5. A summary of the post-processing options is provided in Section 6, and some conclusions are drawn in Section 7. Appendix A contains a list of the variables used in the program and their meaning, and Appendix B lists the entire program.

\section{SIMULATION OF DETECTOR RESPONSE}

Organic scintillators are good candidates for the detection of fast neutrons and photons from fission, and have been applied in a number of measurement systems for the detection of nuclear materials [2]. The output format of the MCNP-PoliMi simulations allows for flexibility in simulating the response of this and other detector types [3-4].

An example of the collision output table from MCNP-PoliMi ver. 1.1 is given in Table 1. Each neutron and photon interaction occurring in user-specified cells is listed in the table, and information about each interaction is reported. Interaction type, target nucleus, energy deposited in the collision, time at which the collision occurred, and position of the collision are among the data reported in the output table. The last column of the table gives the energy of the incident particle before the collision. For example, the first row of the table refers to a $1.6 \mathrm{MeV}$ neutron that interacts via elastic scattering with a carbon nucleus, depositing $339 \mathrm{keV}$ in the collision. The interaction occurred $9.4 \mathrm{~ns}$ after the originating source event, in cell number 9 . 
Table 1. Excerpt from MCNP-PoliMi output file.

\begin{tabular}{|c|c|c|c|c|c|c|c|c|c|c|c|c|c|c|}
\hline $\begin{array}{l}\text { History } \\
\text { number }\end{array}$ & $\begin{array}{l}\text { Particle } \\
\text { number }\end{array}$ & $\begin{array}{l}\text { Projectile } \\
\text { type }^{*}\end{array}$ & $\begin{array}{c}\text { Interaction } \\
\text { type }^{\Delta}\end{array}$ & $\begin{array}{l}\text { Target } \\
\text { nucleus }\end{array}$ & $\begin{array}{c}\text { Cell } \\
\text { number } \\
\text { of } \\
\text { collision } \\
\text { event }\end{array}$ & $\begin{array}{c}\text { Energy } \\
\text { deposited } \\
\text { in } \\
\text { collision } \\
(\mathrm{MeV})\end{array}$ & $\begin{array}{c}\text { Time } \\
\text { (shakes) }\end{array}$ & Collis & $\begin{array}{l}\text { sion pos } \\
(x, y, z)\end{array}$ & sition & $\begin{array}{c}\text { Generation } \\
\text { number }\end{array}$ & $\begin{array}{c}\text { Number } \\
\text { scatterings }\end{array}$ & Code & $\begin{array}{l}\text { Energy } \\
(\mathrm{MeV})\end{array}$ \\
\hline \multicolumn{15}{|l|}{$\ldots$} \\
\hline 151 & 4 & 1 & -99 & 6000 & 9 & 0.33893 & 0.944 & -16.71 & -1.73 & 3.92 & 0 & 0 & 0 & $1.61 \mathrm{E}+00$ \\
\hline 151 & 4 & 1 & -99 & 1001 & 9 & 0.71283 & 1.158 & -15.55 & -4.66 & 2.84 & 0 & 1 & 0 & $1.27 \mathrm{E}+00$ \\
\hline 151 & 20 & 1 & -99 & 1001 & 8 & 0.10313 & 1.464 & 13.4 & 3.95 & -4.48 & 3 & 0 & 0 & $2.61 \mathrm{E}+00$ \\
\hline 151 & 20 & 1 & -99 & 1001 & 8 & 1.20735 & 1.488 & 13.91 & 3.96 & -4.51 & 3 & 1 & 0 & $2.50 \mathrm{E}+00$ \\
\hline 151 & 20 & 1 & -99 & 1001 & 8 & 0.05711 & 1.493 & 13.98 & 3.9 & -4.49 & 3 & 2 & 0 & $1.30 \mathrm{E}+00$ \\
\hline 151 & 20 & 1 & -99 & 1001 & 8 & 0.6134 & 1.64 & 15.9 & 2.76 & -4.28 & 3 & 3 & 0 & $1.24 \mathrm{E}+00$ \\
\hline 151 & 20 & 1 & -99 & 1001 & 8 & 0.42279 & 1.756 & 16.49 & 2.15 & -3.34 & 3 & 4 & 0 & $6.24 \mathrm{E}-01$ \\
\hline 151 & 20 & 1 & -99 & 1001 & 8 & 0.07376 & 1.974 & 17.29 & 2.74 & -2.42 & 3 & 5 & 0 & 2.01E-01 \\
\hline 151 & 20 & 1 & -99 & 1001 & 8 & 0.08552 & 2.297 & 17.55 & 2.64 & -0.89 & 3 & 7 & 0 & $1.14 \mathrm{E}-01$ \\
\hline 164 & 23 & 1 & -99 & 1001 & 9 & 0.22836 & 4.21 & -18.07 & -4.43 & -3.21 & 1 & 3 & 10 & 2.84E-01 \\
\hline 164 & 4 & 2 & 1 & 1 & 8 & 0.11409 & 0.193 & 14.87 & 4.73 & -1.2 & 0 & 1 & 0 & 6.49E-01 \\
\hline
\end{tabular}

The simulation of the detector pulse requires that the energy deposited by neutrons and photons in the detectors be converted into light output by using measured detector response functions. Photons are detected primarily by Compton scattering and the pulse height to energy deposited response is very close to linear

$$
L=E_{\gamma},
$$

where $E_{\gamma}$ is the energy deposited by the photon (MeV) and $L$ is the measured light output (MeVee).

Neutrons are detected primarily by elastic scattering on hydrogen, and the response is non-linear. The neutron and photon pulse-height to energy response functions have been measured [5] for liquid (BC 501) and plastic (BC 420) scintillators. The measured response functions can be fit to a quadratic function as follows:

$$
L=0.0364 \cdot E_{n}^{2}+0.125 E_{n},
$$

for the plastic scintillator, and 


$$
L=0.0350 \cdot E_{n}^{2}+0.1410 E_{n},
$$

for the liquid scintillator where $E_{n}$ is the energy deposited by the neutron on hydrogen (MeV) and $L$ is the measured light output (MeVee). The results of Eq. (1) - (3) are shown in Fig.1. Neutron interactions with carbon are assumed to give a small light output, equal to

$$
L=0.02 E_{n},
$$

where $E_{n}$ is the energy deposited by the neutron on carbon $(\mathrm{MeV})$ and $L$ is the corresponding light output (MeVee).

The detector pulse is generated by the post-processing code by transforming the energy deposited in the individual scattering events into light output using Eq (1), Eq. (4), and Eq. (2) or (3) depending on the scintillator type. The light outputs that occur in an adjustable time window are then added together and compared to a light output threshold. This time window is referred to as "pulse generation time." A typical setting for the pulse generation time is $10 \mathrm{~ns}$ for the scintillators used in the present applications.

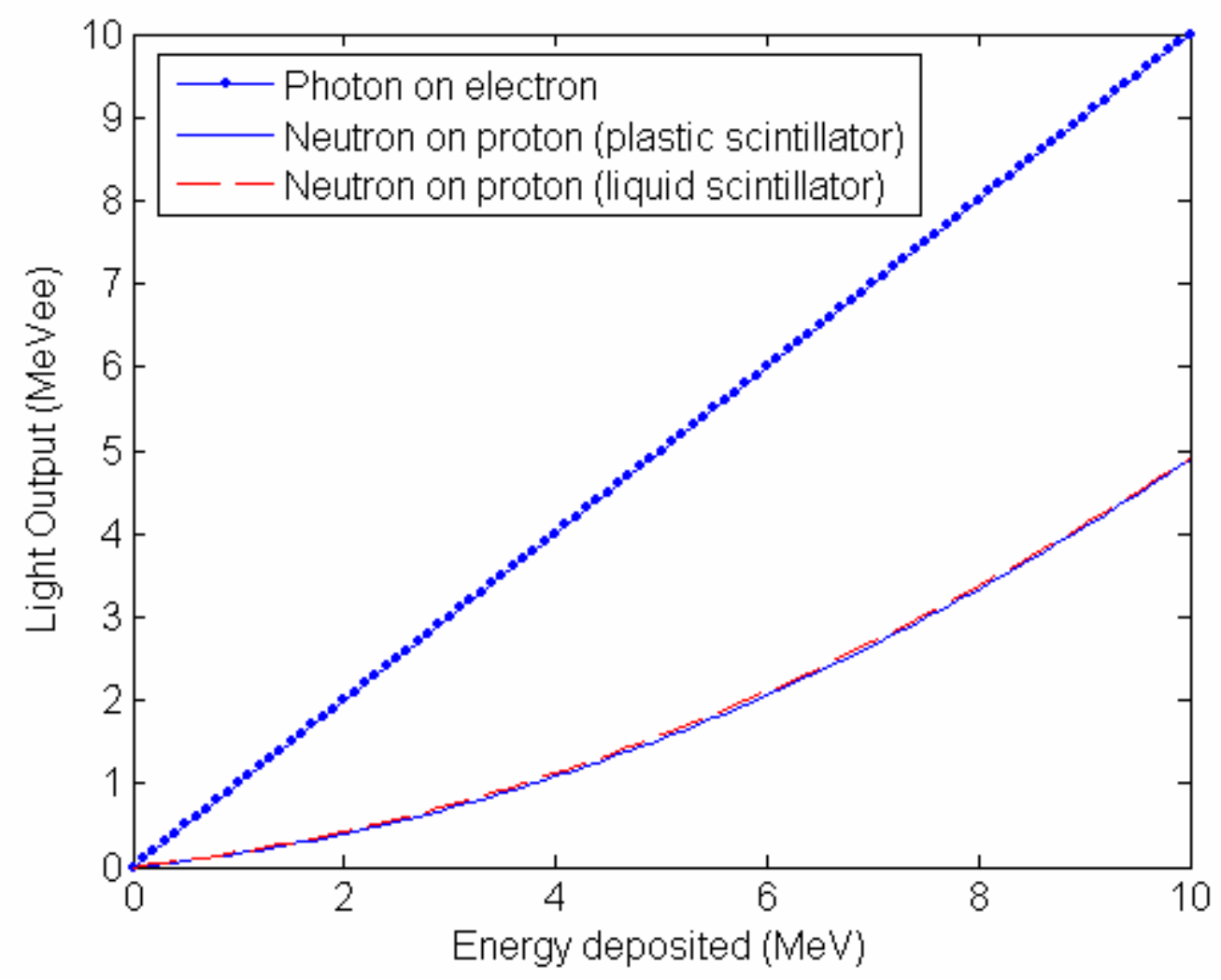

Figure 1. Measured light output functions for plastic (BC 420) and liquid (BC 501) scintillators. 


\section{SIMULATION OF COVARIANCE AND BICOVARIANCE FUNCTIONS}

The post-processing options for the simulation of covariance and bicovariance functions among sequences of events registered by organic scintillators are discussed.

\subsection{Simulation of covariance functions}

The detector response model discussed in Section 2 is used to simulate the time sequences of neutron and photon detection by the scintillators. The time of detection is determined following a source event, which always occurs at time $t=0$. The distribution of two-way coincidence between pairs of sequences over the time delay between counts is given by

$$
R_{x y}\left(\tau_{x y}\right)=E\left[x\left(t_{x}\right) y\left(t_{y}\right)\right]-\bar{x} \cdot \bar{y},
$$

where $\tau_{x y}=t_{y}-t_{x} . \quad R_{x y}$ is known as the covariance between the two sequences of events $x(t)$ and $y(t)$. The product of the means, $\bar{x} \cdot \bar{y}$, yields the rate of accidental coincidence between the two channels. In the simulation, the rate of accidental coincidences is equal to zero because each history evolves independently of all other histories, so the covariance is given, according to Eq. (5), by the expected value of the product of the sequences of events $x(t)$ and $y(t)$. In the simulation, the sequence $x(t)$ (or start sequence) can be given by the source events or by a sequence of detector events. These two options correspond to the measurement of the source-detector covariance or the detector-detector covariance. In the post-processing program, one of these two options is evoked by answering the question 'Do you have a start detector?' If the answer to this question is yes, the detector-detector covariances are computed; otherwise the source-detector covariance is computed.

The post-processing code also performs an analysis of the covariance functions according to particle type (neutron or photon) and generation number (source or induced fission particles). This information is present in the output file in column 3 and 12 of Table 1 , respectively. The variable names corresponding to these covariance functions are listed in Appendix A.

Figure 2 shows the comparison of an MCNP-PoliMi simulation with a measurement performed with a Cf-252 source and a plastic scintillator, having dimensions 7 by 7 by 10 $\mathrm{cm}$, approximately. In the experiment, the Cf-252 source was placed at a distance of $1 \mathrm{~m}$ from the plastic scintillator, and the source-detector covariance was measured. The acquired signature consists of two peaks; the first, sharp peak is given by the gamma rays and the second, broad peak is given by the neutrons. 


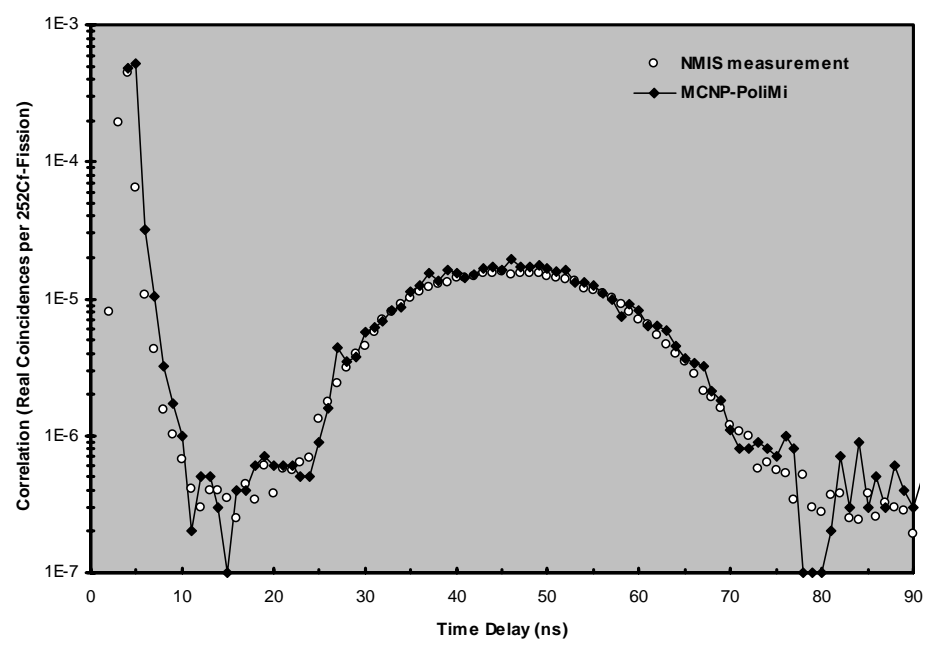

Figure 2. Comparison of Monte Carlo simulation and measurement for a Cf-252 source and detector covariance function.

Inspection of Figure 2 shows that there is very good agreement between the simulation and the measurement.

\subsection{Simulation of bi-covariance functions}

The extension of the covariance function to three sequences of events yields the bicovariance function, or three-way coincidence among three sequences of events:

$$
R_{x y z}\left(\tau_{x y}, \tau_{x z}\right)=E\left[x\left(t_{x}\right) y\left(t_{y}\right) z\left(t_{z}\right)\right]-\bar{x} \cdot R_{y z}\left(\tau_{x z}-\tau_{x y}\right)-\bar{y} \cdot R_{x z}\left(\tau_{x z}\right)-\bar{z} \cdot R_{x y}\left(\tau_{x y}\right)-\bar{x} \cdot \bar{y} \cdot \bar{z} .
$$

This distribution is a function of two delays, $\tau_{x y}=t_{y}-t_{x}$ and $\tau_{x z}=t_{z}-t_{x}$. In our application, the bicovariance function can be computed among the sequences of events in a source and two detectors, or among three detectors. In the first case, a contribution to the bicovariance is made when detections occur in the two detectors as a result of the same source event. In the second case, a contribution is made when all three detectors register a count. The current version of the post-processing code can calculate the bicovariance functions in both these cases. 
Figure 3 shows the sketch of an experiment performed on a plutonium metal shell having mass $4.0 \mathrm{~kg}$, outer radius $6.0 \mathrm{~cm}$ and inner radius $5.35 \mathrm{~cm}$. Further details on the experiments and their simulation are given elsewhere [6-7].

Figure 4 shows the comparison of the measured source-detectors bi-covariance for a plutonium metal shell (98 wt\% Pu-239). Comparison of Figure 4 (a) and (b) shows that the four regions of the bi-covariance are well represented in the simulated signature. Inspection of Figure 4 (a) reveals that there was a 1 ns time offset in the timing alignment of the two detectors used in the measurement.

Contributions to the bi-covariance can be due to neutrons or photons and to particles from the source or particles from induced fission in the fissile material. The information on what type of particle was detected is present in the collision output file. This capability is illustrated in Figures 5 and 6. Figure 5 shows the result of Figure 4 (b) separated into four components according to the particle type that was detected. Experimentally, a split of the bi-covariance according to particle types can be achieved by using liquid scintillators, which have pulse shape discrimination properties.

Figure 6 shows a split of Figure 4 (b) performed according to generation of the particles pairs detected by the scintillators. In our nomenclature, generation zero particles are source particles than reach the detector; later generation particles originate from fission induced in the fissile material.

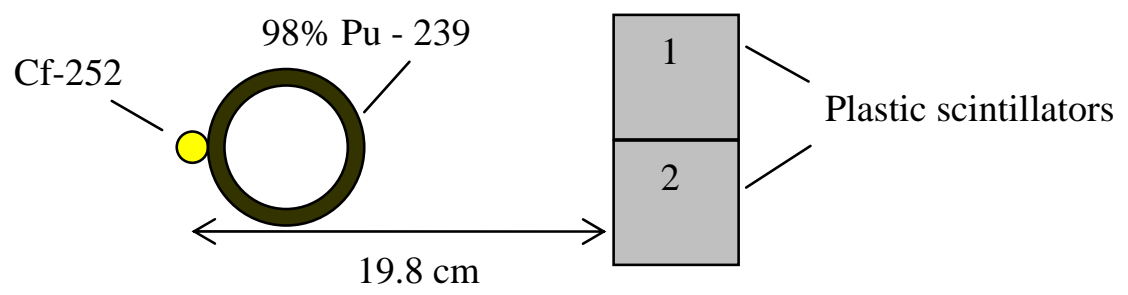

Figure 3. Sketch of the configuration for the simulation of the source-detectors bicovariance function with plutonium sample (not to scale). 


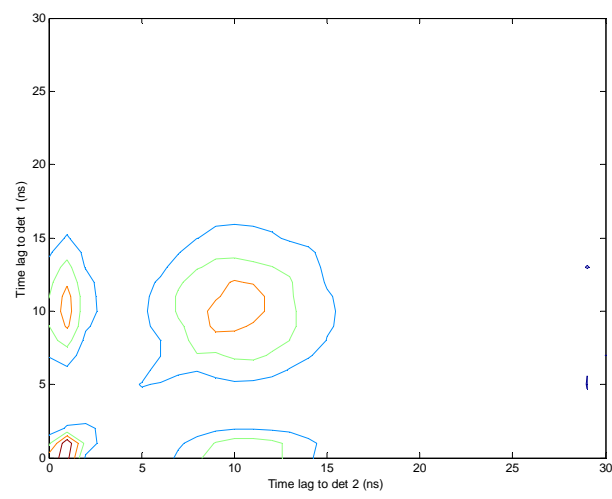

(a)

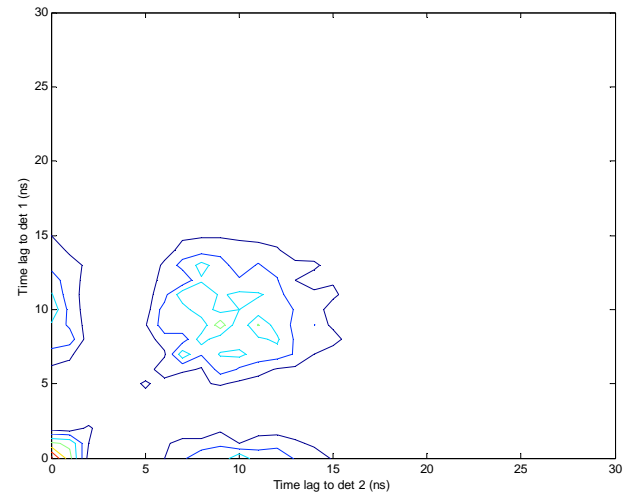

(b)

Figure 4. Source-detectors bi-covariance with plutonium sample: (a) Experimental result and (b) MCNP-PoliMi simulation.
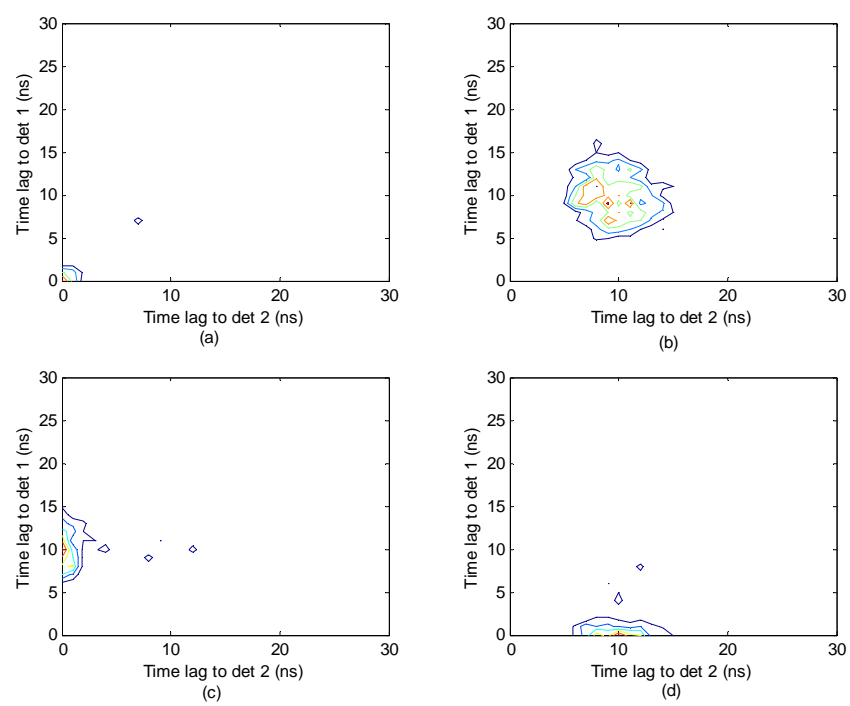

Figure 5. Simulation of source-detectors bi-covariance with plutonium sample: (a) photon - photon pairs, (b) neutron - neutron pairs, (c) neutron - photon pairs, and (d) photon - neutron pairs. 

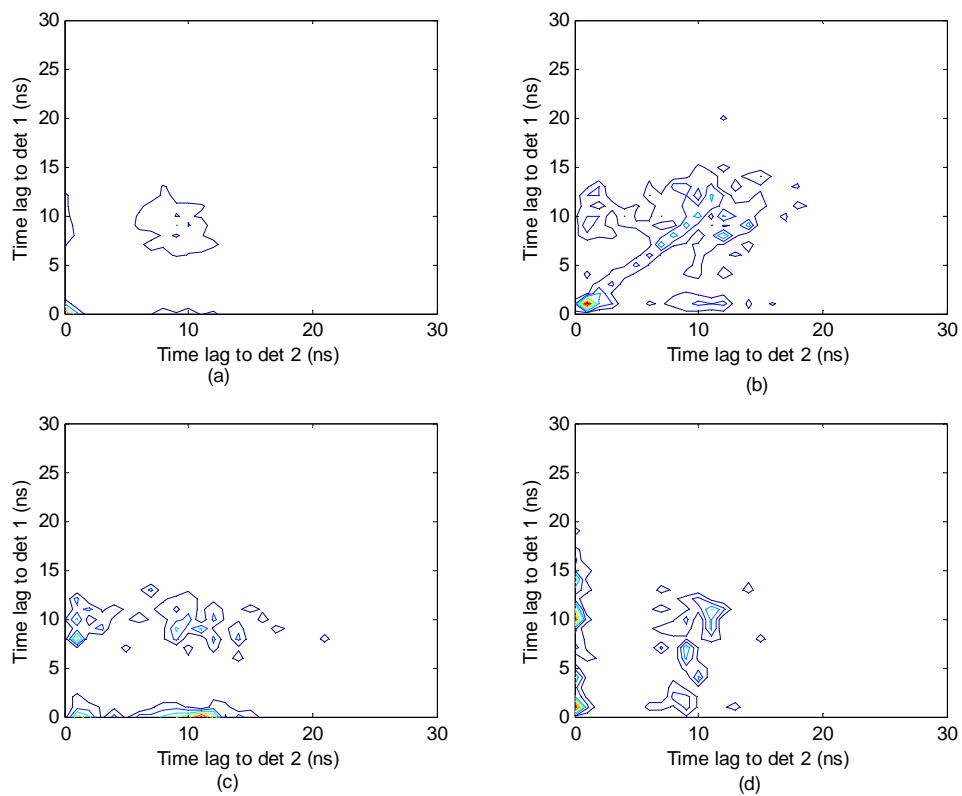

Figure 6. Simulation of source-detectors bi-covariance with plutonium sample: (a) generation zero - generation zero pairs, (b) induced fission - induced fission pairs, (c) generation zero - induced fission pairs, and (d) induced fission - generation zero pairs.

\section{DETECTOR DEAD TIME}

Previous versions of the post-processing code allowed only one detection per detector per history. The current version allows for up to three detections per detector per history, on average $^{1}$. A parameter called "detector dead time" has been included to determine if successive pulses, pertaining to a given history and exceeding the detector threshold, are accepted in the detection sequence registered by the detector. In the experiment, this parameter corresponds to the constant fraction discriminator output pulse time-width. Typically, in our experiments this value lies between 50 and 100 ns.

Figure 7 shows the effect of varying the detector dead time in the simulation of an active measurement on a $3.3 \mathrm{~kg}$ plutonium metal shell (98 wt\% $\mathrm{Pu}-239$ ). The curve with the dots (top curve in Fig. 7) shows the result for a dead time of 0 ns: i.e. all pulses exceeding the threshold are accepted. The bottom curves show the result for a dead time of 5, 20, and 100 ns. There is very little difference in the bottom curves, showing that in this example the occurrence of successive pulses in the detector is rare after a few ns from the first pulse.

\footnotetext{
${ }^{1}$ This parameter can be easily increased as necessary.
} 


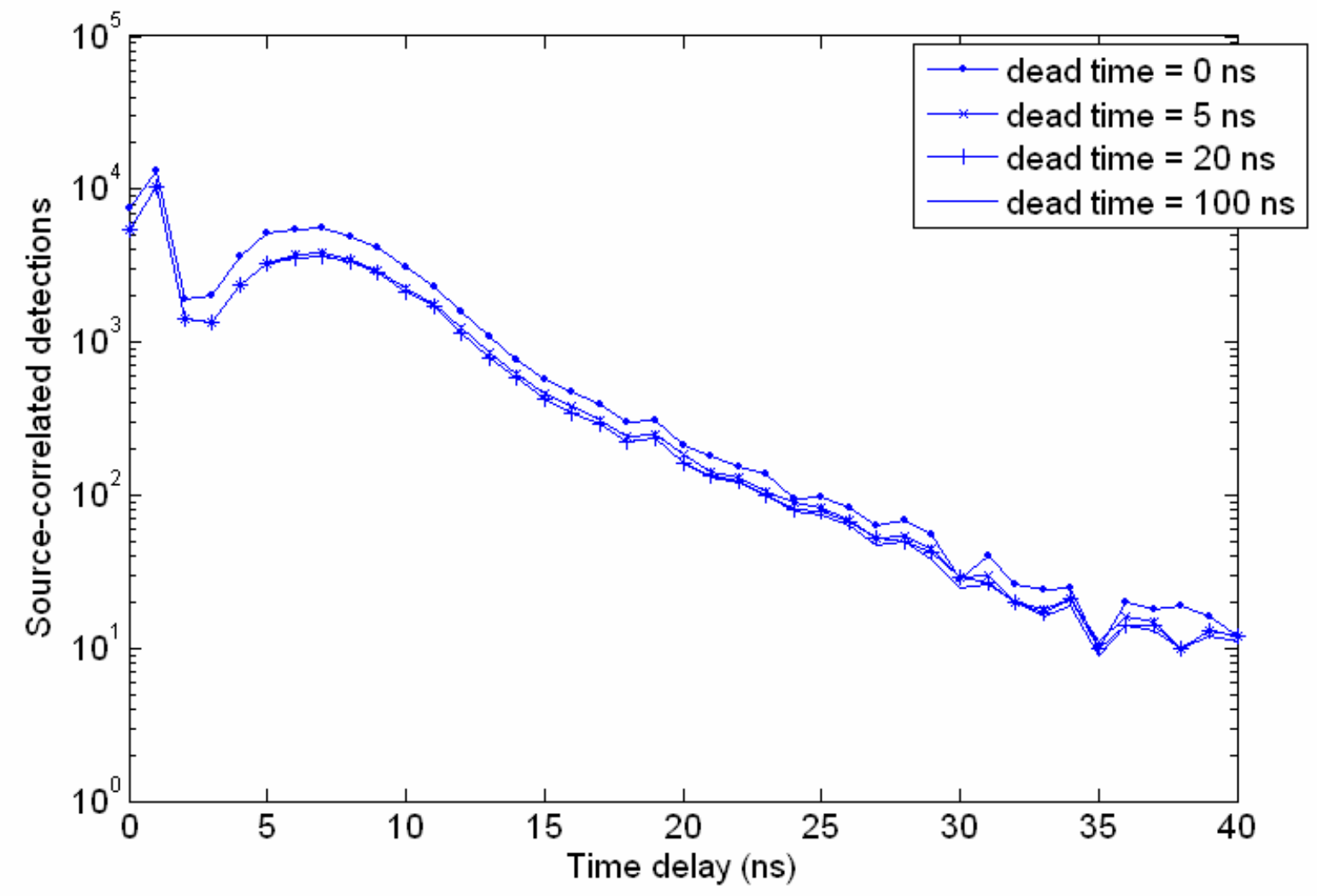

Figure 7. Source-detectors correlation function for $3.3 \mathrm{~kg}$ Pu metal sample and varying detector dead times.

\section{SIMULATION OF DETECTOR MULTIPLETS}

The code simulates the occurrence of multiple pulses in $\mathrm{N}$ detectors within a given time window. The time window can be started by a source event or by a detector event. The time window can be preceded by a pre-delay, time during which the multiplicity counting is dead.

The simulation does not take into account accidentals because each history is initiated by a single source event and evolves independently of any other history, until all the particles pertaining to it are tracked. Figure 8 illustrates the simulation of a source-triggered multiplet (Fig. 8a) and a detector-triggered multiplet (Fig. 8b) registered by $\mathrm{N}$ detectors following a source event. The detection events contribute to the multiplet if they occur in the time window, which begins after the pre-delay. The detections that contribute to the multiplets 
can be given by neutrons or photons ${ }^{2}$. The post-processing code distinguishes between the two particle types.

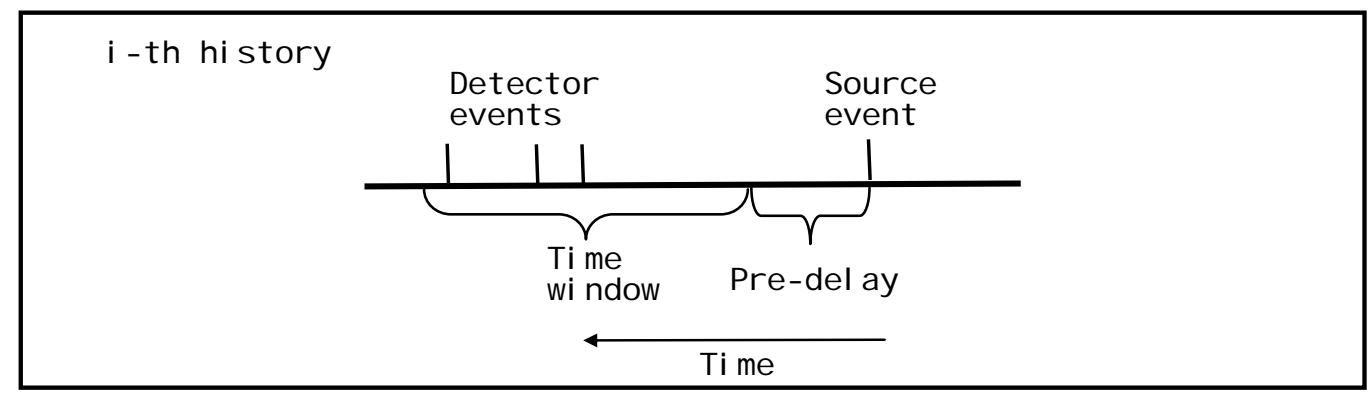

(a)

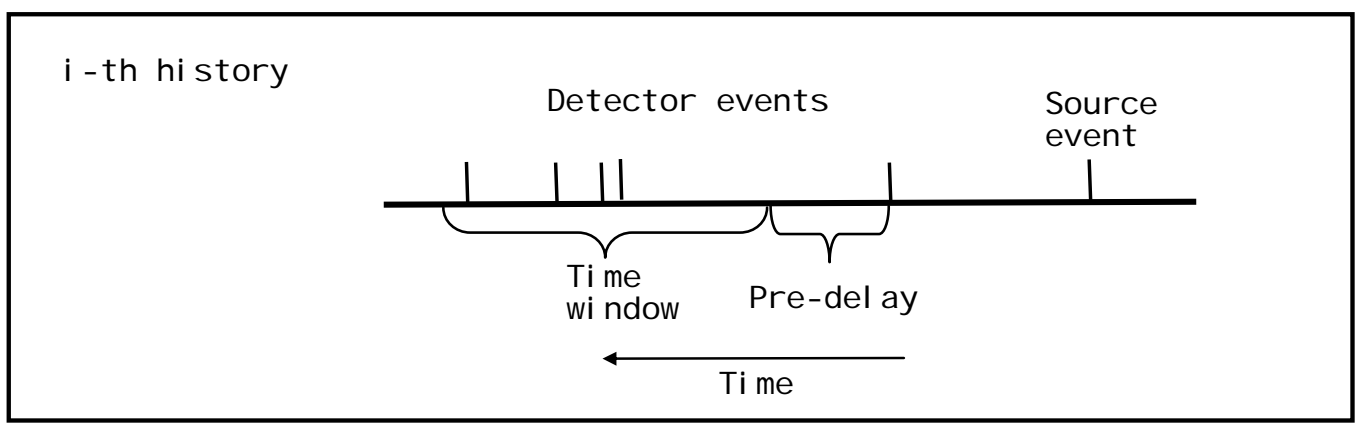

(b)

Figure 8. Illustration of pulse train for multiplet simulation. (a) source-triggered multiplets (b) detector triggered multiplets.

Figure 9 shows the distribution of multiplets registered by two detectors in the active simulation of a $3.3 \mathrm{~kg}$ plutonium shell. The time window was set at $100 \mathrm{~ns}$, and the results are shown for two detector dead times.

\footnotetext{
${ }^{2}$ In some cases, a pulse will result from the combined energy deposition of neutron(s) and photon(s). The occurrence of this event is rare when compared to the occurrence of a pulse generated by only one neutron or photon, interacting multiple times in the detector. In the current classification scheme a pulse is classified on the basis of the particle that contributes the first interaction to the pulse.
} 


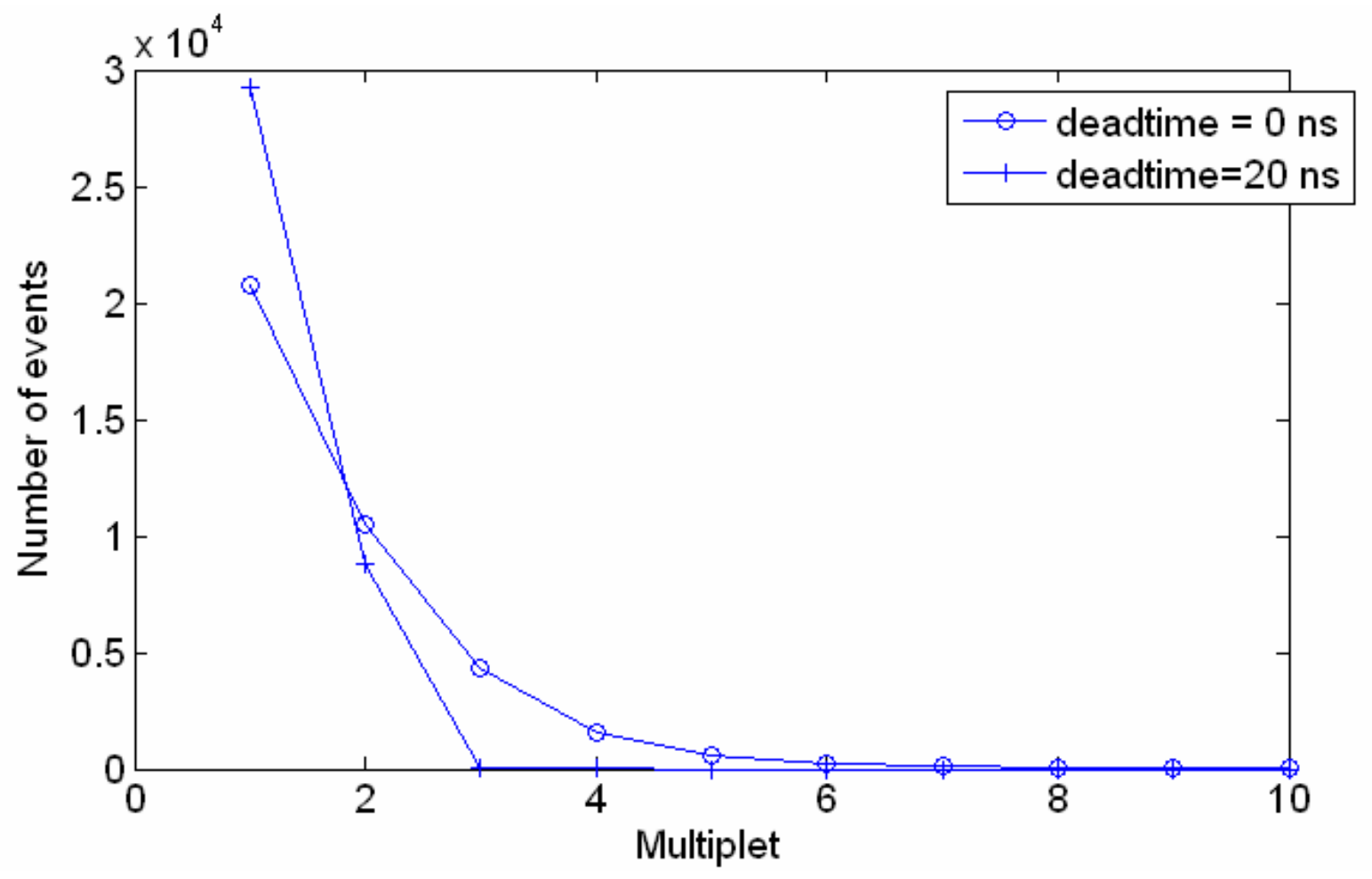

Figure 9. Source-triggered multiplets for $3.3 \mathrm{~kg} \mathrm{Pu}$ metal sample and varying detector dead times. The time window was set to $100 \mathrm{~ns}$.

\section{SUMMARY OF POST-PROCESSING OPTIONS}

Table 2 shows the adjustable parameters in the post-processing code, and indicates where these parameters are set, whether in the post-processing code itself or in the Matlab ${ }^{\mathrm{TM}}$ window. Care should be taken in setting the detector threshold, which can be determined for a given experiment by performing a simple calibration with a gamma source. 
Table 2. Main input parameters in post-processing code.

\begin{tabular}{|c|c|c|}
\hline Adjustable parameters & Possible values & Setting \\
\hline Light output curve & $\begin{array}{l}1 \text { plastic } \\
2 \text { liquid }\end{array}$ & In code \\
\hline Pulse threshold & Threshold in MeVee & In code \\
\hline Pulse generation time & Time in shakes $(1$ shake $=10 \mathrm{~ns})$ & In code \\
\hline Detector dead time & Time in shakes $(1$ shake $=10 \mathrm{~ns})$ & In code \\
\hline Detector numbers & $\begin{array}{l}\text { MCNP-PoliMi cell numbers } \\
\text { of detectors in input file }\end{array}$ & In Matlab ${ }^{\mathrm{TM}}$ window \\
\hline Time for correlation window & Time in ns & In Matlab ${ }^{\mathrm{TM}}$ window \\
\hline Higher order statistics (HOS) & 1 yes & In Matlab ${ }^{\mathrm{TM}}$ window \\
\hline option & 0 no & \\
\hline Multiplicity option & $\begin{array}{l}1 \text { source triggered } \\
2 \text { detector triggered }\end{array}$ & In Matlab ${ }^{\mathrm{TM}}$ window \\
\hline
\end{tabular}

\section{CONCLUSIONS}

This report described the recent additions to the post-processing code used to analyze the MCNP-PoliMi data output file, and serves as a user's manual for the code itself. The new features include the addition of detector dead time and multiplet and bicovariance simulation. A list of the variables used in the post-processor is given in Appendix A. A complete listing of the code itself is given in Appendix B.

\section{REFERENCES}

1. S.A. Pozzi, E. Padovani, and M. Marseguerra, "MCNP-PoliMi: A Monte Carlo Code for Correlation Measurements," Nuclear Instruments and Methods in Physics Research A, 513/3 pp. 550-558, 2003.

2. J. T. Mihalczo, J. A. Mullens, J. K. Mattingly and T. E. Valentine, "Physical description of nuclear materials identification system (NMIS) signatures," Nuclear Instruments and Methods in Physics Research A, 450/2-3, pp. 531-555, 2000. 
3. S.A. Pozzi, J.S. Neal, R.B. Oberer, and J.T. Mihalczo, "Monte Carlo Analysis of Neutron Detection with a BaF2 Scintillation Detector," IEEE Transactions on Nuclear Science, volume 51, number 3, June 2004.

4. S.A. Pozzi, R.B. Oberer, and J.S. Neal, "Analysis of the Response of CaptureGated Organic Scintillators,” 2004 IEEE Nuclear Science Symposium (NSS), October 16-22, 2004, Rome, Italy.

5. S.A. Pozzi, J.A. Mullens, and J.T. Mihalczo, "Analysis of Neutron and Photon Detection Position for the Calibration of Plastic (BC-420) and Liquid (BC-501) Scintillators,” Nuclear Instruments and Methods in Physics Research Section A, 524/1-3 pp. 92-101, 2004.

6. J. K. Mattingly, J. T. Mihalczo, L. G. Chiang, and J. S. Neal, "Preliminary Analysis of Joint RFNC-VNIIEF/ORNL Measurements Performed In Year 2000," Y/LB-16,097, Y-12 National Security Complex, September 2001.

7. S. A. Pozzi, J. K. Mattingly, J. T. Mihalczo, and E. Padovani, "Validation of the MCNP-PoliMi Code for the Simulation of Nuclear Safeguards Experiments on Uranium and Plutonium Metal," Nuclear Mathematical and Computational Sciences: A Century in Review, a Century Anew, Gatlinburg, Tennessee, April 611, 2003, on CD-ROM, American Nuclear Society, LaGrange Park, IL (2003).

\section{APPENDIX A - VARIABLES}

\section{Table I. Postprocessor variables and their meaning.}

\begin{tabular}{cl}
\hline Variable name & Variable meaning \\
\hline 'codes' & Code of reaction generating particle \\
'deadtime' & Detector dead time \\
'gennums' & Particle generation number \\
'idet' & Cell numbers of detectors \\
'idn' & Number of detectors \\
'ilo' & Light output choice \\
'lonc' & Light output neutron on carbon \\
'lonh' & Light output neutron on hydrogen \\
'lop' & Light output photon \\
'multopt' & Multiplicity option \\
'n' & Covariance function (one column per detector) \\
'nc' & Covariance function cross talk events \\
'ncal' & Neutron light output calibration \\
'nch' & Number of collisions in history \\
'ng' & Covariance function induced fission events \\
'nn' & Covariance function neutron events \\
\hline
\end{tabular}




\begin{tabular}{cl}
\hline 'nnc' & Covariance function no cross talk events \\
'np' & Covariance function neutron-photon events \\
'npulses' & Number of detections per detector per history \\
'ns' & Covariance function source events \\
'parnums' & Particle number \\
'partyps' & Particle type \\
'pcal' & Photon calibration \\
'pgt' & Pulse generation time \\
'pn' & Covariance function photon-neutron events \\
'pp' & Covariance function photon-photon events \\
'ptimes' & Sequence of detection times \\
'sdis' & Number of source events \\
'thresh' & Light output threshold \\
'tim' & Time binning \\
'tmax' & Covariance max time \\
'tmin' & Covariance min time \\
'zzz' & PoliMi data output \\
\hline
\end{tabular}




\section{APPENDIX B - MATLAB ${ }^{\mathrm{TM}}$ CODE}

\section{The following is a listing of Matlab program postmain.m, and functions pulsemultip.m and binit.m.}

\section{postmain.m}

\% Loads and analyzes MCNP-POLIMI output file

\% 16 columns of ascii output file have the following information:

\% \# of Start Event, Part \#, Part Type, Reaction type (Ntyn), ZAID collision nucleus, detector cell \#

$\%$ energy rel (MeV), time (shakes), $x, y, z$, wgt, generation \#, \# scatterings, mtp or code, energy of

\% particle prior to present collision

\% note: in the variable names $\mathrm{p}=$ photon; $\mathrm{n}=$ neutron

\% For further information contact Sara Pozzi (pozzisa@ornl.gov)

global pgt lonc ncal pcal thresh deadtime zzz index nch lindex ilo

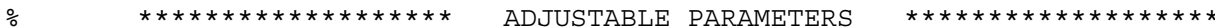

\%ilo=input('Enter your choice for light output: (1) constant (2) lin fit ');

ilo=2; $\quad$ recommended value: ilo=2 for plastic scint. and ilo=3 for liquid

scint.

isep=0; $\quad$ if separate detector responses isep=1

pgt $=0.5 ;$

lonh $=0.16$

lonc $=0.02$

$\%$ pulse generation time in shakes ( 1 shake $=10 \mathrm{~ns}$ )

lop $=1$;

ncal=[lonh 0]; \% parameters for line in calib: neutrons

pcal $=\left[\begin{array}{ll}l o p & 0\end{array} ; \quad\right.$ o parameters for line in calib: photons

if $i l o==2$

end

ncal $=\left[\begin{array}{ll}0.0364 & 0.1250\end{array}\right] ; \%$ parameters for line in calib: neutrons

if $i l o==3$

end

ncal=[0.035 0.1410$] ; \quad \%$ parameters for line in calib: neutrons

thresh=0.187; $\quad$ o selectable threshold for pulse acceptance MeVee units if lop=1

deadtime=50; $\quad$ o detector dead time in shakes ( 1 shake = $10 \mathrm{~ns}$ )

\% note: detector dead time is measured starting from the first

interaction in the pulse

\% multiplicity options

timewindow=51.2; \% time window for multiplicity (shakes; 1 shake = 10 ns)

predelay $=0$; predelay for detection triggered multiplicity

filen=input ('Please enter the file name ','s'); \%file name

ign=input ('Generation analysis ? (0) no (1) yes ');

isc=input ('Separate neutron and photon contributions ? (0) no (1) yes ') ,

idn=input ('Enter number of detectors ');

istar $=0$;

if $i d n>1$

end

istar=input ('Do you have a START detector ? (0) no (1) yes ');

istop=idn-istar

if istop $<1$ end error('Must have at least one STOP detector')

idet $=$ zeros $(1, i d n)$ :

if istar $==1$

disp(' Insert the cell numbers for detectors.')

disp(' The START detector must be the first one entered.')

elseif istar $=0$ else disp(' Insert the cell numbers for STOP detectors. ')

end error ('Invalid istar entry')

for $j=1: i d n$ cell=input ( ['Enter cell number for detector ', num2str(j), ' ']) , 


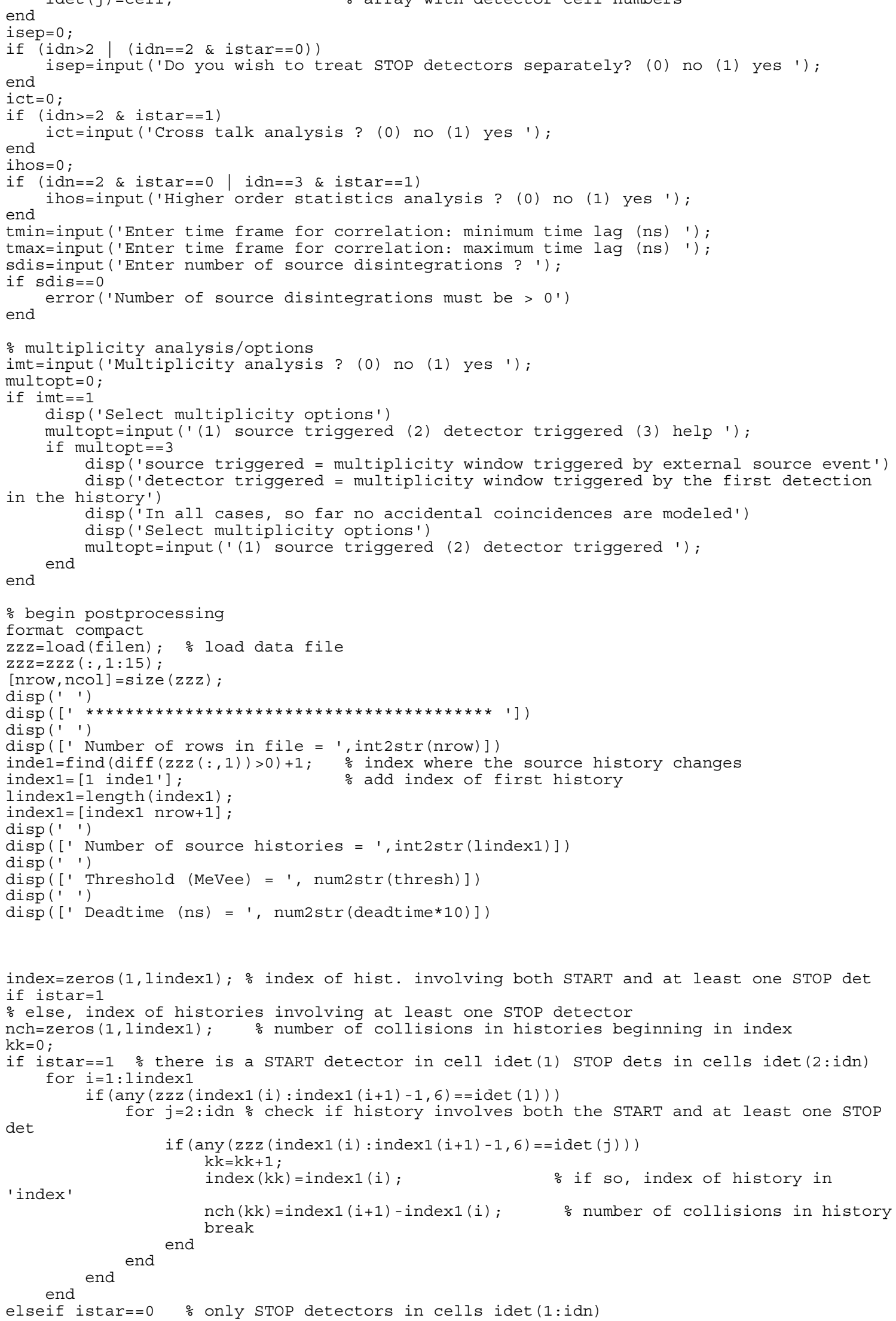




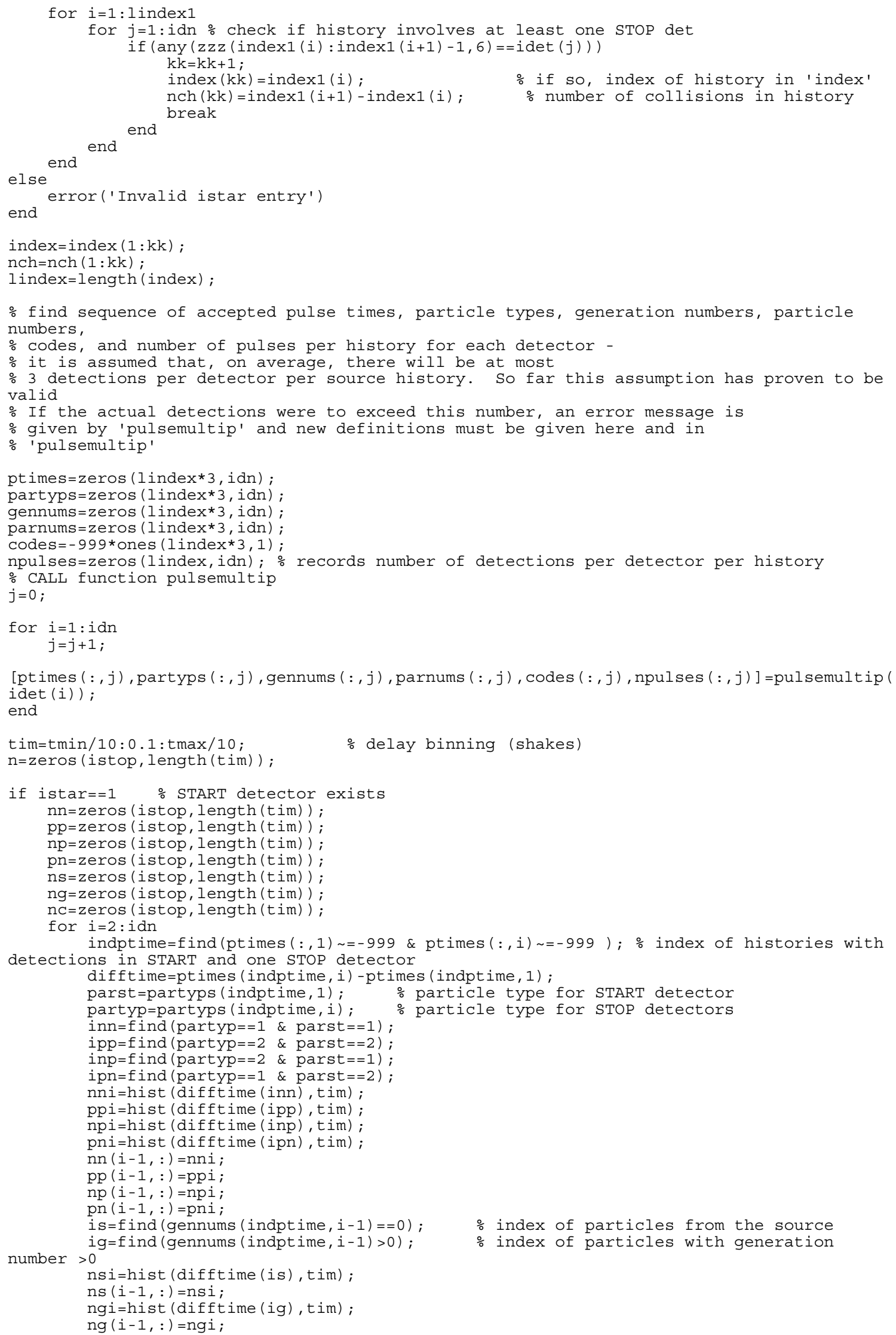




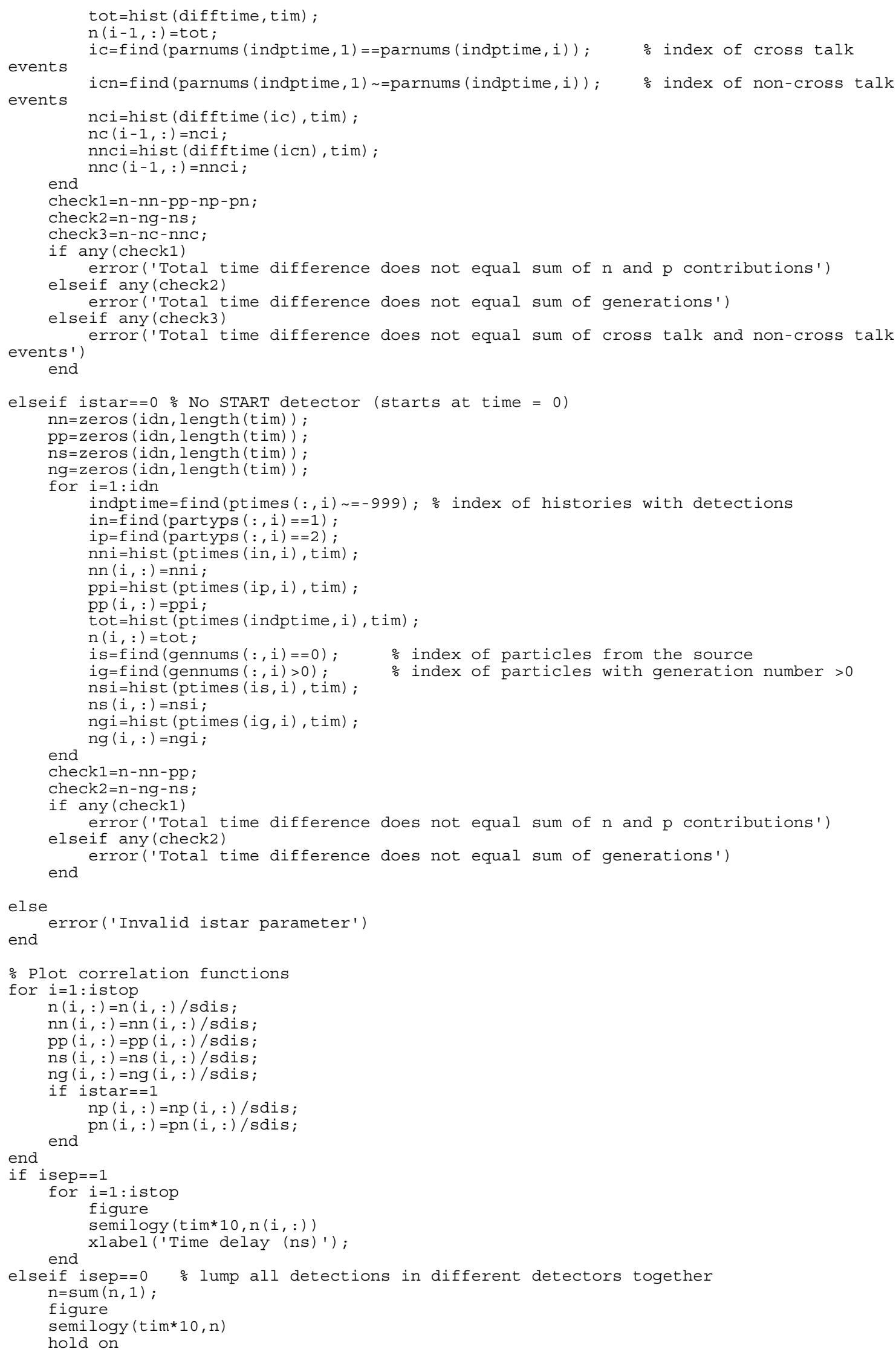




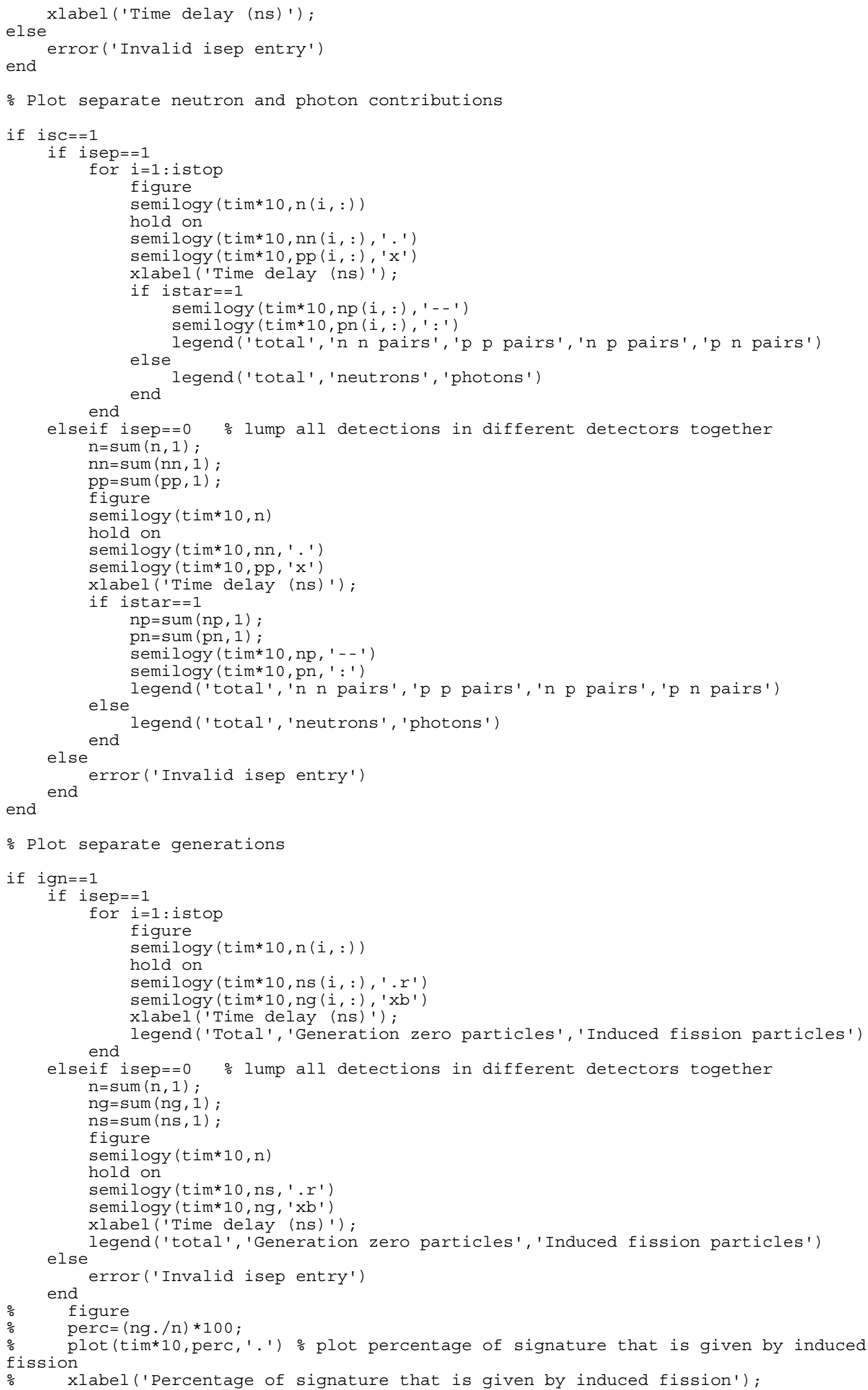




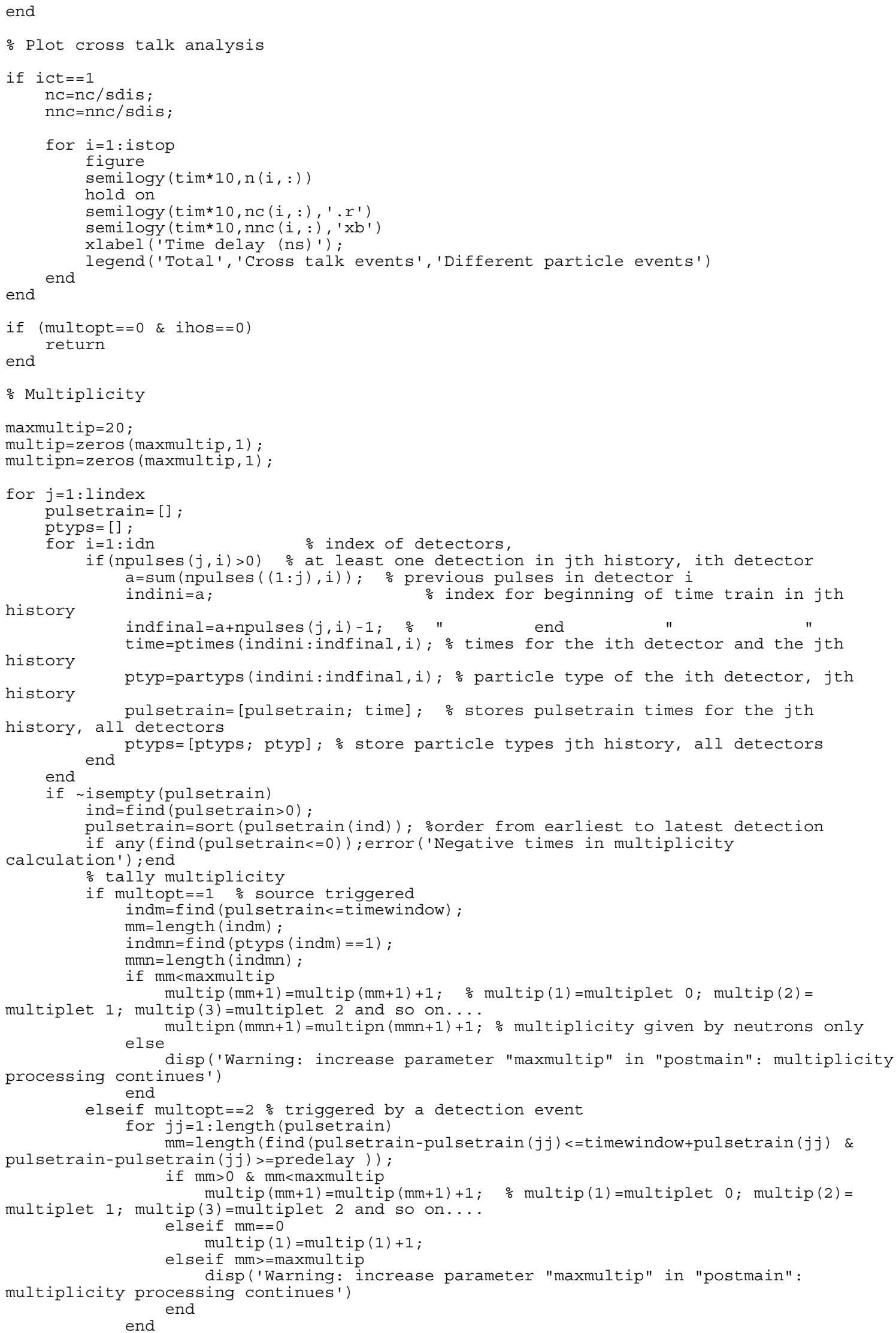


multip(1)=sdis-sum(multip); $\%$ set multiplicity of 0's to number of source events - sum of any multiplet

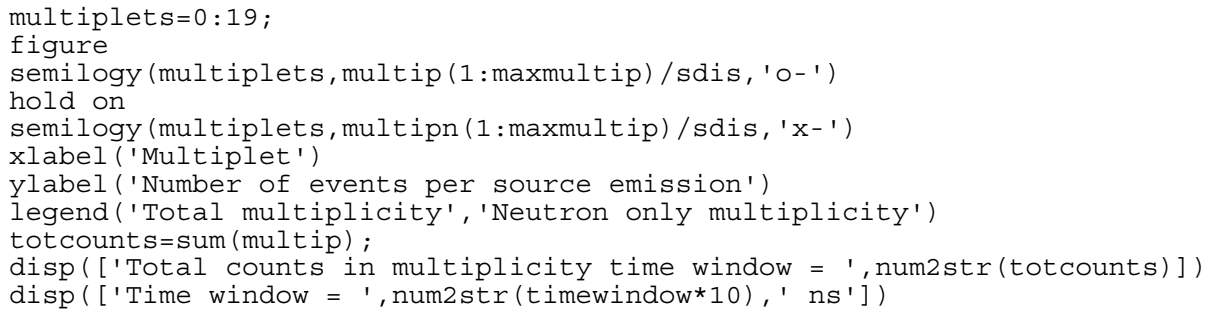

check1=mathos-mathosnn-mathospp-mathosnp-mathospn; check2 =mathos-mathosss - mathosgg-mathossg-mathosgs; if any (check1) 
figure : plot separate photon and neutron contributions

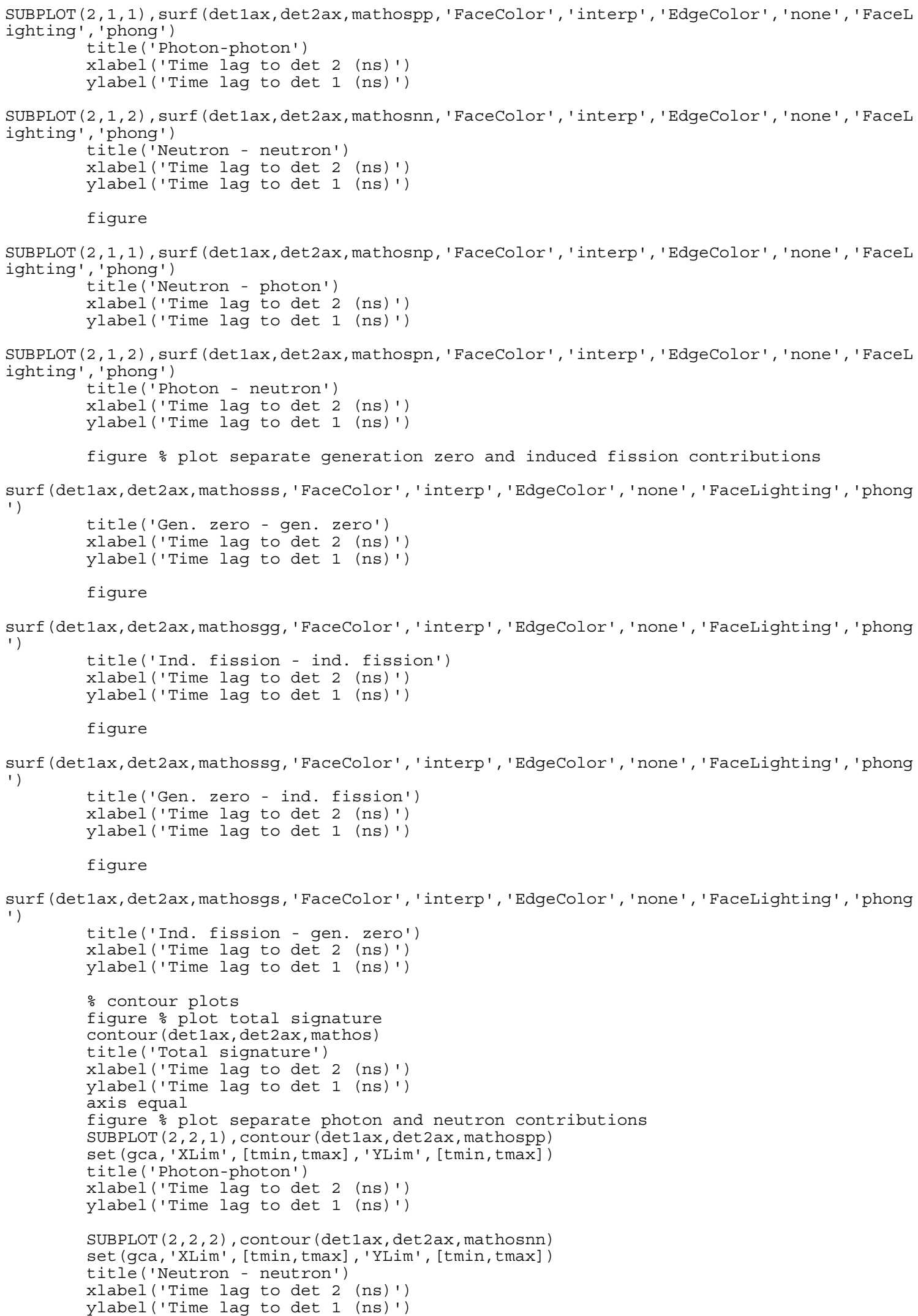


SUBPLOT $(2,2,3)$, contour (det1ax, det 2 ax, mathosnp)

set (gca, 'XLim', [tmin,tmax] , 'YLim', [tmin, tmax])

title ('Neutron - photon')

xlabel('Time lag to det 2 (ns)')

ylabel ('Time lag to $\operatorname{det} 1$ (ns)')

SUBPLOT $(2,2,4)$, contour (det1ax, det2ax, mathospn)

set (gca, 'XLim', [tmin, tmax], 'YLim', [tmin, tmax])

title('Photon - neutron')

xlabel ('Time lag to det 2 (ns)')

ylabel('Time lag to $\operatorname{det} 1$ (ns)')

axis equal

figure \% plot separate generation zero and induced fission contributions $\operatorname{SUBPLOT}(2,2,1)$, contour (detlax, det2ax, mathosss)

set (gca, 'XLim', [tmin,tmax] , 'YLim', [tmin, tmax])

title('Gen. zero - gen. zero')

xlabel('Time lag to $\operatorname{det} 2$ (ns)')

ylabel ('Time lag to $\operatorname{det} 1$ (ns)')

SUBPLOT $(2,2,2)$, contour (detlax, det2ax, mathosgg)

set (gca, 'XLim', [tmin, tmax], 'YLim', [tmin, tmax])

title('Ind. fission - ind. fission')

xlabel ('Time lag to det 2 (ns)')

ylabel ('Time lag to det 1 (ns)')

$\operatorname{SUBPLOT}(2,2,3)$, contour (detlax, det2ax, mathossg)

set (gca, 'xLim', [tmin, tmax], 'YLim', [tmin, tmax])

title('Gen. zero - ind. fission')

xlabel ('Time lag to $\operatorname{det} 2$ (ns)')

ylabel('Time lag to $\operatorname{det} 1$ (ns)')

SUBPLOT $(2,2,4)$, contour (det1ax, det2ax, mathosgs)

set (gca, 'XLim', [tmin, tmax], 'YLim', [tmin, tmax])

title('Ind. fission - gen. zero')

xlabel('Time lag to $\operatorname{det} 2$ (ns)')

ylabel ('Time lag to $\operatorname{det} 1$ (ns)')

end

return 


\section{pulsemultip.m}

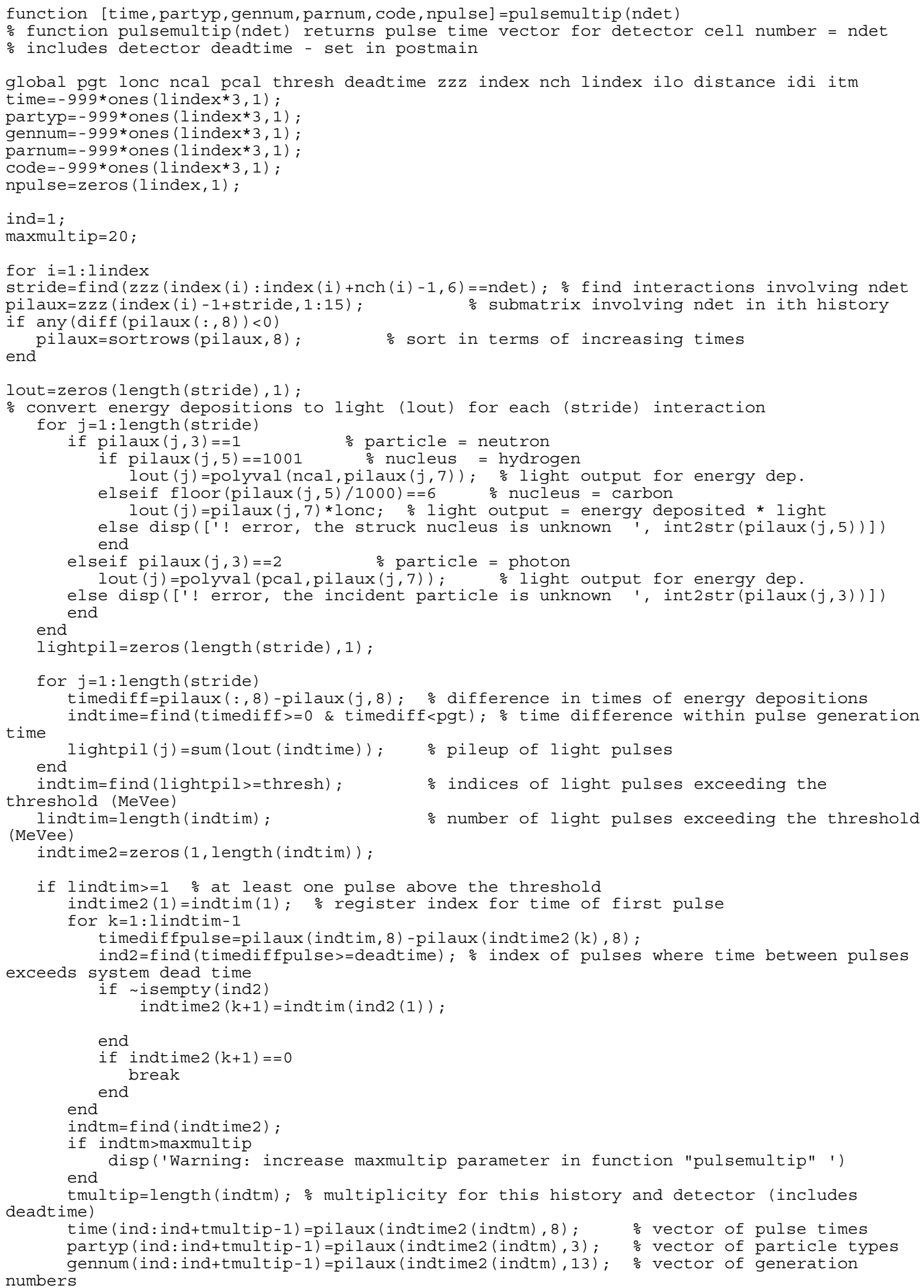




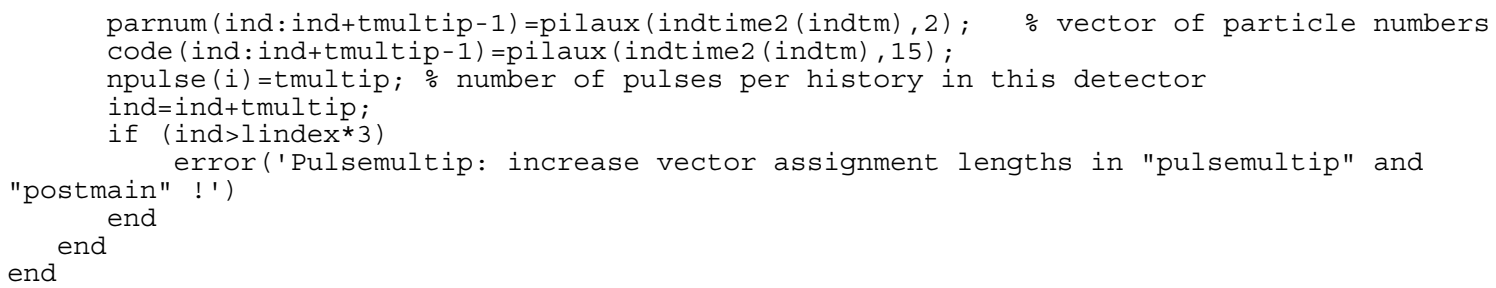

\section{binit.m}

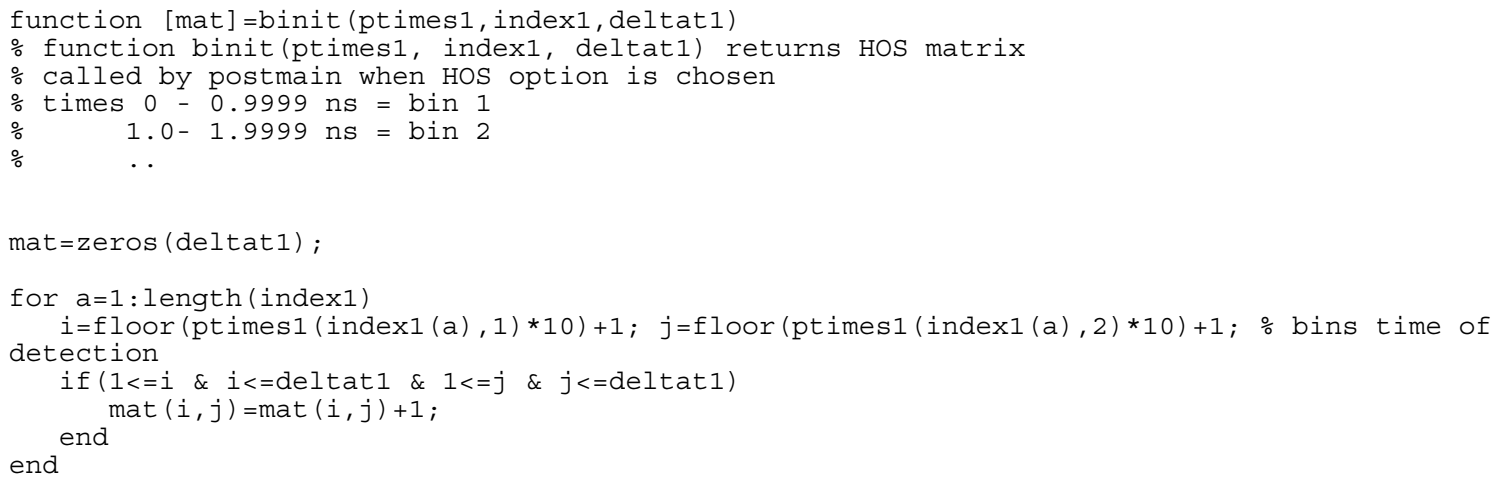

